\title{
Violencia contra la mujer: conocimiento y actitud del personal médico del Instituto Mexicano del Seguro Social, Morelos, México
}

\author{
Pablo Méndez-Hernández, M en $C{ }^{(1)}$ Rosario Valdez-Santiago, MA, (2) Leonardo Viniegra-Velázquez, MC, (3) \\ Leonor Rivera-Rivera, M en $C_{,}{ }^{(4)}$ Jorge Salmerón-C astro, Dr en C. ${ }^{(5)}$
}

Méndez-Hernández P,Valdez-Santiago R, Viniegra-Velázquez L, Rivera-Rivera I, Salmerón-Castro J.

Violencia contra la mujer: conocimiento y actitud del personal médico del Instituto Mexicano del Seguro Social, Morelos, México. Salud Pública Méx 2003;45;472-482. El texto completo en inglés de este artículo está disponible en: http://www.însp.mx/salud/index.html

\section{Resumen}

Objetivo. Evaluar la actitud afectiva, cognoscitiva y conductual del personal médico del Instituto Mexicano del Seguro Social del estado de Morelos, México, hacia la identificación y canalización de mujeres maltratadas que acuden a consulta médica, así como identificar las barreras institucionales y de la práctica médica que dificultan el manejo dentro de los servicios de salud de las mujeres maltratadas. Material y métodos D e septiembre a diciembre de 1999 se realizó un estudio transversal mediante un cuestionario de autoaplicación que se proporcionó a 269 médicos y médicas generales, especialistas y estudiantes de pre y posgrado que laboran en 30 unidades de salud, de primer y segundo nivel de atención, del Instituto Mexicano del Seguro Social del estado de Morelos. El instrumento se diseño para evaluar el nivel de conocimiento sobre violencia doméstica y la actitud del personal médico hacia la atención, en la consulta, de mujeres maltratadas. Se construyó un índice de cono cimiento, además de un análisis multivariado de los datos. Resultados. El $90 \%$ de los entrevistados nunca ha recibido capacitación en violencia contra la mujer. La actitud

\author{
Méndez-Hernández P,Valdez-Santiago R, \\ Viniegra-Velázquez L, Rivera-Rivera I, \\ Salmerón-Castro J. \\ Violence against women: knowledge and attitudes \\ of healthcare providers at the Mexican Institute \\ of Social Security in Morelos, Mexico. \\ Salud Pública Méx 2003;45;472-482. \\ The English version of this paper \\ is available at: http://www.insp.mx/salud/index.html
}

\begin{abstract}
A bstract
Objective. To asses the affective, cognitive, and behavioral attitudes of healthcare providers at the Mexican Institute of So cial Security (MISS) in Morelos, Mexico; to identify the institutional and medical practice bar riers that hinder screening and reference of battered women. Material and Methods. A cross-sectional study was conducted between September and December 1999. A self-administered questionnaire was applied to 269 general practitioners, specialists, and pre- and postdoctoral students working in 30 primary and secondary level of healthcare units in Morelos State. The data collection instrument was designed to assess healthcare providers' knowledge of and attitudes towards domestic violence during medical office visits. A knowledge index was constructed and analyzed using multivariate regression methods. Results $\mathrm{N}$ inety percent of healthcare providers had never received training on violence against women. Healthcare providers' affective and cognitive attitudes after receiving training on the subject matter were more favorable compared to those with no training. Favorable attitudes were directly related to the number of
\end{abstract}

Trabajo financiado por el Consejo N acional de Ciencia y Tecnología (Becario: 126013), y por el Estudio de cohorte de trabajadores del Instituto Mexicano del Seguro Social.

(1) Departamento de Ciencias de la Salud, Universidad Autónoma de Tlaxcala,Tlaxcala, México.

(2) Centro de Investigación en Sistemas de Salud, Instituto N acional de Salud Pública (IN SP), Cuernavaca, Morelos, México.

(3) Unidad de Investigación Educativa. Centro Médico N acional siglo XXI, Instituto Mexicano del Seguro Social (IMSS), México, D F, México.

(4) Centro de Investigación en Salud Poblacional, IN SP, Cuernavaca, Morelos, México.

(5) Unidad de Investigación Epidemiológica y en Servicios de Salud, IMSS, Cuernavaca, Morelos, México.

Fecha de recibido: 21 de mayo de 2001 • Fecha de aprobado: 15 de abril de 2002 Solicitud de sobretiros: Pablo Méndez Hernández. Universidad Autónoma de Tlaxcala, Departamento de Ciencias de la Salud. Boulevard Emilio Sánchez Piedras s/n, Colonia Centro,Tlaxcala,Tlaxcala, México. Correo electrónico: pmendez@ garza.uatx.mx y pmendezh@ hotmail.com 
afectiva y cognoscitiva del personal médico que recibió capacitación sobre el tema fue más favorable hacia la identificación y atención de mujeres maltratadas que llegan a consulta médica, en comparación con los que no la recibieron. Sobre el nivel de conocimiento acerca del tema, $21 \%$ de los entrevistados mostró un nivel bajo, $63 \%$ medio y $16 \%$ alto. En el personal médico, con nivel de conocimiento medio y alto, se observó 2.1 y 6 veces - respectivamente- una actitud afectiva más favorable respecto a la identificación y canalización de casos de mujeres maltratadas en comparación con el personal con nivel de conocimiento bajo. Las médicas mostraron 2.3 veces mejor actitud para identificar y canalizar mujeres maltratadas. El personal médico con interés por capacitarse en el tema mostró 7.6 veces mejor actitud que el personal no interesado. Conclusiones. El personal médico evaluado mostró habilidades insuficientes para identificar y manejar adecuadamente los casos de mujeres maltratadas que atienden en su consulta. Este estudio evidencia la necesidad de capacitar y sensibilizar al personal médico sobre la materia; se recomienda empezar con los médicos generales y familiares, quienes demostraron estar más dispuestos a capacitarse, en comparación con los de especialidades clínicas e incorporar esta capacitación en los planes de estudio de medicina, enfermería, trabajo social, psicología y áreas afines a la salud pública. El texto completo en inglés de este artículo está disponible en: http:// www.insp.mx/salud/index.html

Palabras clave: mujeres maltratadas; violencia doméstica; actitud; conocimiento; médicos; México training sessions. Most participants (63\%) showed a moderate degree of knowledge on the subject, whereas $21 \%$ were slightly knowledgeable and $16 \%$ were highly knowledgeable. Medical perso nnel with a moderate or high level of knowledge were 2.1 and 6 times more likely, respectively, to have favor rable attitudes than those with a low degree of knowledge. Female physicians showed more favorable attitudes towards identifying and referring battered women. Medical personnel interested in further training on the subject of violence against wo men were 7.6 times more likely to show favorable attitudes than personnel not interested on the subject. Conclusions $\mathrm{H}$ ealthcare providers were not sufficiently able to assess and manage battered women. $G$ eneral and family practitioners were more interested in being trained, as compared with specialist physicians. Training on violence against women should be included in graduate medical, nursing, social service, psychology, and other public health areas. The English version of this paper is available at: http://www.insp.mx/salud/index.html

Key words: battered women; domestic violence; attitudes; aw areness; physicians; Mexico a violencia contra la mujer constituye un probleL ma social y de derechos humanos que repercute de manera importante en la salud y bienestar de las mujeres mexicanas y de otras regiones del mundo. A pesar de que la violencia es una causa significativa de morbilidad y mortalidad femenina, sólo hasta hace algunos años se ha visualizado como un problema de salud pública. ${ }^{1}$

Aunque la prevalencia de la violencia contra la mujer suele estar subestimada, ${ }^{2,3}$ en México la prevalencia de violencia doméstica reportada, tanto en zonas rurales como urbanas, oscila entre 30 y $60 \%$., Sin embargo, las cifras registradas en las instituciones de salud, así como en las instancias de procuración de justicia representan sólo la punta del iceberg, ya que cap-

\footnotetext{
* Venguer T, Fawcett G, Vernon R, Pick S. Violencia doméstica: un marco conceptual para la capacitación del personal de salud. México, DF: Population Council, INOPAL III, 1998;24:7-52. Documento interno de trabajo.
}

tan sólo las situaciones de extrema violencia o aquellas en las que las víctimas deciden denunciar, lo cual ocurre en la minoría de los casos. ${ }^{5,6}$

La violencia hacia las mujeres es un problema importante, entre otras razones, por los daños que ocasiona en la salud, a saber: a) lesiones físicas; b) trastornos psicológicos ${ }^{7}$ y psicosomáticos; ${ }^{8} \mathrm{c}$ ) trastornos ginecológicos y daños a la salud reproductiva, y d) riesgo de adquirir enfermedades de transmisión sexual, inclusive el VIH-SIDA. ${ }^{9} 10$ Durante el embarazo el abuso representa riesgos significativos, tanto para la madre como para el producto, entre los que destacan bajo peso al nacer, escaso aumento de peso en la madre, parto prematuro, así como infecciones y anemia. ${ }^{11,12, *}$

\footnotetext{
* Instituto Mexicano de Investigación de la Familia y Población A.C. (IMIFAP). La violencia doméstica: lo que el personal de salud debe saber. México, DF: IMIFAP, 1998. Folleto de difusión.
} 
El Banco Mundial en 1993 estimó que las mujeres en edad reproductiva pierden $20 \%$ de años de vida saludable (AVISA) a causa de la violencia. ${ }^{13}$ En la Ciudad de México, Lozano y colaboradores reportaron que $38 \%$ de los AVISA perdidos entre las mujeres se deben a la violencia que se ejerce en su contra, y que este problema ocupa el tercer lugar de importancia entre el total de problemas de salud que afectan a la mujer en edad reproductiva. ${ }^{14}$

El reconocimiento social de este problema ha impactado los códigos civil y penal en la República Mexicana, ${ }^{15,16}$ y dio la pauta para que la Secretaría de Salud normara la atención de los casos de violencia familiar, a raíz de la publicación de la Norma Oficial Mexicana para la prevención y control de la violencia familiar. ${ }^{17}$

Pese a los avances logrados hasta el momento aún prevalece una permisividad social alrededor de la violencia hacia las mujeres, lo cual impide que el problema se detecte y se atienda de manera oportuna, siendo este el principal obstáculo para su prevención y control.* Entre los elementos que dificultan la identificación y manejo de casos de violencia en los servicios de salud podemos señalar los que se refieren a los prestadores de servicios, al sistema de salud, y la reacción de temor de las propias víctimas.

En el caso de las mujeres maltratadas, a diferencia de otras víctimas de crímenes violentos, es común que tengan dificultades para expresar el origen de sus lesiones. Entre las razones que subyacen a esta conducta podemos señalar que se sienten avergonzadas y culpables de la violencia que sufren. Otra razón de su silencio son las amenazas que reciben de su agresor, las cuales incluyen las de muerte en contra de ellas o de sus hijos. ${ }^{18,10}$

En relación con los profesionales de la salud, los estudios realizados en este sector demuestran las siguientes situaciones: a) existe un desconocimiento generalizado en el personal médico de los procedimientos para la identificación, atención y mecanismos de referencia en los casos de violencia hacia las mujeres, en especial en relación con la violencia por parte de la pareja, ${ }^{12,19}$ b) en ocasiones, aunque el personal médico desea proporcionar algún tipo de atención u orientación a sus pacientes, puede verse atrapado en las limitaciones del propio sistema de salud; ${ }^{20} \mathrm{c}$ ) el personal médico percibe como un problema la responsabilidad legal que implica el registrar los casos de violencia, lo que limita su registro; d) las características propias de la práctica médica limitan su intervención en los casos de violencia hacia las mujeres ${ }^{21}$ y e) finalmente, se ha observado que existen barreras de carácter personal que limitan a los profesionales de la salud. Entre éstas resaltan la incomodidad para abordar la violencia entre sus pacientes, las concepciones sexistas que culpabilizan a las mujeres de la violencia que sufren, y el temor de los médicos(as) de ofender a sus pacientes al preguntar sobre el tema. ${ }^{22}$

En este sentido, las instituciones de salud son un punto clave para la detección y prevención de los daños a la salud de las mujeres maltratadas, ya sea en la consulta regular o en los servicios de urgencias, pues en estos últimos se ha identificado que $20 \%$ de los casos de demanda de atención fueron a consecuencia de la violencia doméstica, la mayoría hacia las mujeres $(72 \%) .{ }^{23}$ Además, se ha demostrado que $70 \%$ de las mujeres maltratadas esperan que el personal médico sea quien inicie el diálogo en torno al abuso, y cuando se les pregunta con empatía, libre de juicios y en un clima de confianza, sí expresan la situación de abuso en la que viven.*

La elevada prevalencia de la violencia contra la mujer, y sus secuelas médicas y psicológicas, justifican plenamente su detección rutinaria. ${ }^{24}$ No identificar el abuso como tal interfiere con el diagnóstico real, encareciendo los servicios de salud en términos de tiempo y dinero, debido a las consultas subsecuentes y al tratamiento de las secuelas.

Con base en lo anterior, el presente estudio tuvo por objetivo evaluar la actitud afectiva, cognoscitiva y conductual del personal médico del Instituto Mexicano del Seguro Social (IMSS), del estado de Morelos, México, hacia la identificación y canalización de mujeres maltratadas que acuden a consulta médica, así como identificar las barreras institucionales y de la práctica médica que dificultan el manejo de estas mujeres dentro de los servicios de salud.

\section{Material y métodos}

Durante septiembre a diciembre de 1999 se realizó un estudio transversal en médicos y médicas que trabajan en el IMSS del estado de Morelos.

Participaron en el presente estudio 269 médicos (as) internos(as) de pregrado, residentes de especiali-

\footnotetext{
* Fawcett G, Venguer T, Vernon R, Pick S. Detección y manejo de mujeres víctimas de violencia doméstica: desarrollo y evaluación de un programa dirigido al personal de salud. México, DF: Population Council, INOPAL III, 1998;26:7-22. Documento interno de trabajo.
} 
dad y médicos(as) generales de las 27 unidades de salud del primer y segundo nivel de atención.

Los médicos(as) fueron visitados en su área de trabajo y se les invitó a participar en el estudio; a quienes aceptaron participar se les proporcionó un cuestionario de autoaplicación para que fuera llenado dentro o fuera del turno laboral, dándoles la oportunidad de regresarlo hasta una semana después. Luego, cuando los participantes entregaron el cuestionario, recibieron un folleto de información sobre violencia contra la mujer, y una lista con direcciones de los servicios disponibles en el estado de Morelos hacia donde podían canalizar a las mujeres maltratadas que atendieran posteriormente en su consulta.

El cuestionario fue construido para cumplir los objetivos de la presente investigación; además, se retoman algunas preguntas hechas previamente a personal médico del Hospital General de México, de la Secretaría de Salud (SSA) y del Departamento del Distrito Federal.‡ Además, se recupera la guía de entrevista a profundidad, aplicada en 1997 al personal médico del primer nivel de atención que atienden mujeres embaradas en los Servicios de Salud de Morelos (SSM). ${ }^{12} \mathrm{El}$ cuestionario fue probado previamente en médicos(as) de la SSA y del IMSS y consta de seis secciones: la primera, se refiere a información sociodemográfica. La segunda, evaluó el nivel de instrucción previa del personal médico sobre violencia hacia la mujer. La tercera, la actitud cognoscitiva de los médicos sobre el tema; para medirla se construyó un índice de conocimiento mediante 12 preguntas, considerando el nivel de conocimiento de acuerdo con el número de preguntas acertadas: nivel bajo, a los participantes que respondieron adecuadamente entre 1 y 4 preguntas; medio, entre 5 y 8 , y alto, entre 9 y12. La cuarta sección se refirió a los factores institucionales y de la práctica profesional que el personal médico percibe como barreras para identificar y canalizar casos de mujeres maltratadas que acuden a consulta. La quinta sección evaluó la actitud afectiva del personal médico respecto de: a) interés por identificar los casos de mujeres maltratadas (actitud para identificar casos), b) interés por

\footnotetext{
* Instituto Mexicano de Investigación de la Familia y Población A.C. (IMIFAP). La violencia doméstica: lo que el personal de salud debe saber. México, DF: IMIFAP, 1998. Folleto de difusión.

‡ Fawcett G, Venguer T, Vernon R, Pick S. Detección y manejo de mujeres víctimas de violencia doméstica: desarrollo y evaluación de un programa dirigido al personal de salud. México, DF: Population Council, INOPAL III, 1998;26:7-22. Documento interno de trabajo.
}

canalizar adecuadamente los casos de mujeres maltratadas (actitud para canalizar casos), c) interés por capacitarse en el tema, y d) actitud afectiva general (actitud general), la cual se evaluó al incorporar los tipos de actitud antes mencionados. La sexta sección permitió conocer -de manera indirecta- la actitud conductual ante los casos de mujeres maltratadas, ya que sólo se les preguntó lo que hacen durante su desempeño laboral. Además, permitió conocer si el personal médico tenía experiencias personales (propias o de personas cercanas) respecto a la violencia en la pareja.

\section{Medición de actitud}

La variable dependiente fue la actitud (afectiva, cognitiva y conductual). Por lo que partimos de la definición de Allport, ${ }^{25}$ que cuenta con gran aceptación, y se toma como base para la sistematización de las representaciones acerca de la actitud: "La actitud consiste en un estado mental y neural de disposición organizado a través de la experiencia, que ejerce una influencia directiva o dinámica sobre la conducta del individuo ante todos los objetos y situaciones con los que se relaciona". ${ }^{26}$ Sin embargo, a pesar de que esta definición es bastante completa, otros autores integran el aspecto sociopsicológico; es decir, su dependencia del grupo (o colectividad) y, en consecuencia, de las normas y valores, los cuales son expresión de la estructura socioeconómica del régimen social y expresados, así mismo, por el grupo; de esta manera, podemos asumir que la actitud constituye también una forma de orientación del proceder de las personas, dependiente de la norma grupal, y tiene la propiedad de reflejarse en el comportamiento del individuo, orientarlo, regularlo y guiarlo en determinado sentido, pudiéndose distinguir en toda actitud su objeto, dirección e intensidad. ${ }^{27}$

En la estructura de las actitudes se distinguen tres componentes: a) cognitivo, se refiere a las ideas o información que tiene un individuo respecto del objeto de actitud; b) afectivo, se relaciona con el sentimiento de simpatía o antipatía, respecto del objeto de actitud, y c) conductual, indica el comportamiento del individuo respecto del objeto de actitud.

En el presente estudio se evaluaron los componentes cognitivo, afectivo y conductual de las actitudes y, debido a que la relación entre el componente afectivo y el conductual no siempre es lineal, se implantaron circunstancias que ayudaran a obtener mediciones confiables de lo afectivo: a) introduciendo nuevas cogniciones $\mathrm{y} b$ ) ofreciendo un ambiente de privacidad $y$ confianza a los individuos que expresan sus opiniones y creencias. $^{28}$ 


\section{A nálisis estadístico}

Se realizó análisis univariado, estimando medias y proporciones para ver la distribución de las variables de interés y describir las características generales. Posteriormente, se efectuó un análisis bivariado utilizando prueba de ji cuadrada, y modelos de regresión logística para obtener estimadores de razón de momios (RM) con intervalos de confianza (IC) de 95\%. Para controlar las posibles variables confusoras se utilizó análisis de regresión logística múltiple. ${ }^{29}$ Los datos fueron capturados en el programa FOX PRO versión 6 y analizados mediante el programa estadístico STATA, versión 6 .

\section{Resultados}

De los 488 médicos y médicas del IMSS de Morelos se contactó a $440(90 \%)$, de los cuales 269 (61\%) respondieron el cuestionario. Las características de quienes no respondieron fueron similares a los que sí lo hicieron, en relación con edad y sexo. Sin embargo, por zona laboral* se observó menor respuesta en el Hospital Regional de Zona 1 (HRZ 1) de Cuernavaca, en comparación con las tres unidades restantes $(p<0.001)$. Se exploraron las características sociodemográficas y el tipo de especialización médica del personal entrevistado, las cuales se ilustran en el cuadro I; como se puede apreciar la mayoría son hombres (64\%), con 42 años en promedio (rango de edad 22-63) y con pareja (79\%). En cuanto al tipo de especialización se pudieron observar diferencias importantes, por ejemplo, los médicos generales, familiares y ginecobstetras mostraron una mayor tasa de respuesta en comparación con los estudiantes ${ }^{\ddagger}$ y demás médicos especialistas ${ }^{\S} p=0.042$ y $p=0.031$, respectivamente.

En relación con la instrucción previa que el personal médico tenía sobre la violencia contra la mujer, casi 90\% de los participantes informaron no haber recibido capacitación sobre este tema. Respecto de los participantes que sí recibieron alguna instrucción, más de la mitad sólo recibió una, la mayoría fueron médicos familiares en comparación con los especialistas de otras

\footnotetext{
* La delegación Morelos del IMSS está dividida en cuatro zonas laborales: HRZ 1 en Cuernavaca, la Unidad Médica Familiar (UMF) 20 en Cuernavaca, el HGZ 7 en Cuautla y el HGZ 5 en Zacatepec.

‡ Médicos internos de pregrado y médicos residentes de especialidad.

$\S$ Medicina de urgencias, cirugía general, pediatría, medicina interna, psiquiatría, traumatología entre otras especialidades y subespecialidades.
}

\section{Cuadro I \\ Características sociodemográficas del personal mÉdico del Instituto MeXicano del Seguro Social, Morelos, México, 1999}

\begin{tabular}{lcc} 
Variables & Frecuencia & M edia (rango) \\
Edad en años & 269 & $2(22-63)$ \\
\hline Años de práctica médica & 269 & $16(0-37)$ \\
& & Porcentaje \\
Sexo & & \\
Femenino & 97 & 36 \\
\hline Masculino & 172 & 64 \\
Estado civil & & \\
Con pareja & 212 & 79 \\
\hline Sin pareja & 57 & 21 \\
$\begin{array}{l}\text { Categoría del médico } \\
\text { Especialistas en medicina familiar }\end{array}$ & 86 & 32 \\
\hline Médicos generales & 54 & 20 \\
\hline Especialistas en otras áreas & 81 & 30 \\
\hline Médicos con posgrado & 35 & 13 \\
\hline Estudiantes de pregrado y especialidad & 13 & 5 \\
Turno laboral & & \\
Turno matutino & & \\
\hline Turno vespertino & 108 & 40 \\
\hline Jornada acumulada & 75 & 28 \\
\hline $\begin{array}{l}\text { Lugar de residencia } \\
\text { Zona urbana }\end{array}$ & 86 & 32 \\
\hline Zona rural & & \\
\hline & 196 & 73 \\
\hline
\end{tabular}

áreas $(p<0.001)$. El personal que no tenía pareja recibió por lo menos una clase sobre el tema en comparación con el personal con pareja $(p<0.001)$. Cuando relacionamos la actitud general del personal médico respecto a la violencia y el número de capacitaciones que recibieron, encontramos que la actitud del personal que por lo menos recibió una instrucción fue más favorable en relación con el que no la recibió $(p<0.04)$. La actitud general de quienes recibieron de 2 a 4 capacitaciones fue relativamente más favorable, en comparación con aquellos que sólo recibieron una $(p=0.07)$. El mayor número de capacitaciones se impartieron a nivel de posgrado, ${ }^{*}$ en comparación con pregrado ( $p=0.036)$ y en el personal femenino, en comparación con el masculino $(p=0.04)$.

\footnotetext{
* Personal médico con diplomado, maestría y/o doctorado.
} 
Respecto al conocimiento que el personal médico tenía sobre el tema, $21 \%$ mostró nivel de conocimiento bajo, $63 \%$ medio y $16 \%$ alto; con esto se observa mayor nivel de conocimiento entre las médicas, en comparación con los médicos $(p=0.04)$. Otras diferencias importantes fueron identificadas entre quienes recibieron por lo menos una capacitación sobre el tema, en comparación con quienes no lo hicieron $(p=0.02)$; en quienes tienen posgrado, en relación con quienes tienen especialidad ( $p=0.04$ ); en quienes realizan de manera intencional preguntas para identificar mujeres maltratadas, en contraste con quienes no preguntan $(p<0.001)$, $\mathrm{y}$ en el personal médico sin pareja $(p<0.05)$.

Cuando se preguntó al personal médico acerca de los daños a la salud secundarios a la violencia, 70\% respondió acertadamente respecto de los daños físicos, $58 \%$ respecto de los psicológicos, mientras que sólo $41 \%$ respecto de los sexuales. Los médicos familiares y generales respondieron más acertadamente, en comparación con otros especialistas ( $p<0.05$, en ambos casos).

Aunque más de $90 \%$ de los entrevistados estuvo de acuerdo en que la violencia repercute de manera importante sobre la salud de las mujeres, y que el personal médico es el ideal para identificar y canalizar los casos, sólo $46 \%$ mostró una actitud general favorable ante el problema. Esta discordancia podría estar relacionada con las barreras institucionales y las referentes a la práctica médica que los participantes refieren como obstáculos para identificar, y canalizar los casos de las mujeres maltratadas. En el cuadro II se puede apreciar la proporción de médicos que señalaron estos obstáculos, entre los que resaltan la falta de protocolos de detección y manejo de casos (29\%), el carácter priva-

\section{Cuadro II}

Factores que el Personal médico percibe COMO BARRERAS PARA IDENTIFICAR Y CANALIZAR LOS CASOS DE MUJERES MALTRATADAS. Instituto Mexicano del Seguro Social, Morelos, México, 1999

Factores institucionales
$\begin{aligned} & \text { Falta de protocolos de detección y manejo de casos } \\ & \text { No se ha legitimado la participación del médico }\end{aligned}$
$\begin{aligned} & \text { desde el punto de vista legal e institucional } \\ & \text { Falta de tiempo en la consulta }\end{aligned}$
$\begin{aligned} & \text { Factores de la práctica médica } \\ & \text { El carácter privado de la violencia }\end{aligned}$
$\begin{aligned} & \text { Consideran difícil identificar y manejar los casos } \\ & \text { durante el desempeño de la práctica médica }\end{aligned}$

do de la violencia $(26 \%)$ y la falta de legitimación del médico en la intervención de este tipo de situaciones (24\%).

Las variables que se asocian con la actitud general y la actitud para identificar y canalizar casos -tanto en la prueba ji cuadrada como en los modelos de regresión logística multivariados- (cuadros III y IV) fueron, entre otras, el nivel de conocimiento sobre la violencia y el interés por capacitarse en este tema.

Se evaluó también -mediante regresión logística múltiple- la actitud afectiva del personal médico para canalizar los casos de mujeres maltratadas por su pareja, y se encontró que la única variable significativa fue el sexo, es decir, las mujeres mostraron 2.1 veces mejor actitud para canalizar los casos de violencia, en comparación con los hombres, IC 95\% 1.18-4.47, $(p=0.04)$.

Se exploró si alguna vez habían identificado algún caso de violencia hacia la mujer, $78 \%$ respondió afirmativamente. Además, con relación a cuántos casos habían identificado en los últimos tres meses, $68 \%$ respondió haber detectado solamente entre 1 y 2 . Tanto médicos familiares como generales identificaron mayor número de casos que los especialistas en otras áreas. Por otro lado, $70 \%$ consideró que la mujer maltratada es quien debe dar la pauta para que el médico pueda preguntarle acerca de su problema de violencia, y sólo la mitad consideró que la violencia hacia las mujeres es un problema frecuentemente atendido en la práctica médica.

Respecto al tipo de conducta que el personal médico realiza para identificar casos, $68 \%$ hace preguntas

\section{Cuadro III \\ Actitud afectiva general del personal médico DEL IMSS, MORELOS, EN RELACIÓN CON SU CONOCIMIENTO EN EL TEMA Y SU INTERÉS por CAPACITARSE EN ÉL. Instituto MeXicano del Seguro Social, Morelos, México, 1999}

\begin{tabular}{lrrr} 
& \multicolumn{3}{c}{ Actitud afectiva del personal médico } \\
\cline { 2 - 5 } Variables independientes & Razón de momios & $p$ & IC $95 \%$ \\
Nivel de conocimiento medio* & 2.10 & 0.052 & $0.99-4.47$ \\
\hline Nivel de conocimiento alto* & 6.05 & 0.001 & $2.20-16.75$ \\
\hline Género $^{\ddagger}$ & 2.30 & 0.007 & $1.25-4.20$ \\
\hline Interés por capacitarse en el tema $^{\S}$ & 7.26 & 0.010 & $1.61-32.68$
\end{tabular}

Modelo ajustado por edad, estado civil y categoría del médico

* El grupo de referencia es el nivel de conocimiento bajo

* El grupo de referencia son los varones

$\S$ El grupo de referencia son los participantes que mencionaron no tener interés en capacitarse 


\section{Cuadro IV \\ Actitud afectiva del personal médico del IMSS, \\ MoReLOS, RESPECTO DE IDENTIFICAR CASOS DE MUJERES MALTRATADAS DE ACUERDO CON SU NIVEL DE CONOCIMIENTO E INTERÉS POR CAPACITARSE en el tema. Instituto Mexicano del Seguro Social, Morelos, México, 1999}

\begin{tabular}{lccc} 
& \multicolumn{2}{c}{$\begin{array}{c}\text { Actitud afectiva del personal médico } \\
\text { para identificar casos } \\
\text { de mujeres maltratadas }\end{array}$} \\
\cline { 2 - 5 } Variables independientes & 2.15 & 0.040 & $1.03-4.47$ \\
\cline { 2 - 5 } Nazón de momios & P & IC 95\% \\
\hline Nivel de conocimiento medio* & 4.03 & 0.004 & $1.55-10.51$ \\
\hline Nivel de conocimiento alto* & 3.35 & 0.038 & $1.06-10.53$
\end{tabular}

El modelo fue ajustado por edad, estado civil, sexo y categoría del médico

* El grupo de referencia es el nivel de conocimiento bajo

₹ El grupo de referencia son los participantes que mencionaron no tener interés en capacitarse

para identificar el maltrato en mujeres sólo cuando sospechan que se trata de un caso, $6 \%$ lo hace de manera rutinaria y $26 \%$ nunca lo hace. Después de identificar un caso de violencia, el personal médico refiere adoptar las siguientes conductas: a) 38\% platica con las pacientes, tratando de ayudarlas; b) $32 \%$ proporciona tratamiento médico, y c) $25 \%$ refieren los casos a instituciones o personas que les brinden atención especializada.

Respecto a las experiencias personales de violencia en los entrevistados, $21 \%$ refieren haber sido víctimas de algún tipo de violencia por parte de su pareja (49\% psicológica, 27\% física, 13\% económica y 9\% sexual), siendo más frecuente la violencia en contra de las mujeres $(p=0.04)$. El 16\% refiere ser autor de violencia hacia su pareja (58\% psicológica, 32\% física, $6 \%$ económica y $4 \%$ sexual), no existiendo diferencia por sexo. A su vez, $44 \%$ del personal menciona conocer alguna persona cercana a su círculo social con este problema.

\section{Discusión}

Una de las limitaciones del estudio está dada por el tamaño de la muestra, el cual se ve reflejado en la amplitud de los intervalos de confianza obtenidos. Esta situación puede explicarse por la escasa disposición del personal médico para participar en estudios con esta temática, principalmente por parte de especialis- tas y subespecialistas. Esta situación es en sí misma un hallazgo importante que coincide con estudios previos sobre el problema, ${ }^{11,12}$ y constituye un reto metodológico a considerar para quienes se interesen en la investigación en el tema. Entre las razones que explican esta negativa del personal médico podemos señalar la falta de interés sobre la violencia hacia las mujeres, la incomodidad que experimentan al tocar el tema, además de las exigencias propias de la práctica clínica. ${ }^{24}$ Otra razón es la inaccesibilidad a ciertas áreas hospitalarias en donde se encuentran los médicos especialistas.

En este sentido, la falta de participación cuando es mayor en cierto grupo de individuos puede ocasionar subestimación o sobre estimación de las asociaciones obtenidas. ${ }^{30}$ En este caso, existe la probabilidad de que la diferencia de actitud que mostraron los médicos especialistas y subespecialistas (menos favorable), en comparación con los médicos generales y familiares, ginecobstetras, médicos con posgrado y las médicas, pudiera haber sido más importante.

El primer hallazgo que queremos resaltar es que cerca de $90 \%$ del personal médico del IMSS, Morelos, no tiene instrucción alguna sobre el tema; entre quienes sí recibieron alguna capacitación, la mayoría la adquirió por iniciativa propia, mientras que el resto lo hizo de manera obligatoria durante su formación, y la mayoría de las capacitaciones fueron en un nivel de posgrado. Este desconocimiento generalizado sobre el problema es la variable que mejor predice la actitud general y la actitud para identificar en la consulta médica casos de mujeres maltratadas; este hallazgo es consistente con los estudios realizados en el país ${ }^{12,31}$ y a escala internacional. Por ejemplo, Ferris ${ }^{19}$ encontró, en una muestra nacional de 963 médicos familiares en Canadá, que la mayoría de éstos demandaban más educación y capacitación sobre el problema, por considerar que no tienen los elementos suficientes para poder enfrentar la situación con sus pacientes.

Otro aspecto explorado es la relación entre el número de capacitaciones sobre violencia y la identificación, por el personal médico, de signos y síntomas. En nuestro estudio, encontramos que quienes han recibido mayor capacitación pueden identificar una mayor cantidad de signos y síntomas, en comparación de aquellos que no la tienen. Esto es consistente con lo reportado por Parsons y colaboradores, ${ }^{32}$ quienes encuestaron a médicos ginecobstetras en los Estados Unidos de América (EUA), para conocer sus métodos y actitudes hacia la identificación de la violencia doméstica entre sus pacientes; de éstos, 34\% reportó no tener capacitación suficiente. En este estudio se identificó que los médicos con mayor entrenamiento sobre 
el tema estuvieron más propensos a identificar la violencia en sus pacientes.

Por otro lado, resulta evidente considerar que la mayoría del personal médico rara vez sospecha violencia en sus pacientes, por lo que no les hacen preguntas sobre este tema en la mayoría de casos, ${ }^{*}$ y sólo sospechan en aquellos casos que son muy obvios pues las mujeres acuden a los servicios con las lesiones producidas por la violencia física. En este sentido, podemos afirmar que no se cubren las exigencias de identificación, manejo y canalización de las mujeres maltratadas que acuden a los servicios de atención a la salud.

Respecto al número de casos que el personal médico identifica, Fawcett ${ }^{20}$ reportó que $70 \%$ del personal médico del Hospital General de México (SSA) y del Departamento del Distrito Federal identificaron de 1 a 5 casos en un mes; Reid y Glasser ${ }^{21}$ encontraron que $56 \%$ de su muestra ha identificado más de 10 casos en toda su vida laboral. En nuestro estudio, $68 \%$ reportó haber identificado desde ninguno hasta dos casos en tres meses. En las tres situaciones el número de casos es insuficiente ya que, de acuerdo con el promedio de pacientes que los médicos del IMSS atienden por turno, y a la prevalencia reportada en los estudios sobre el problema, el personal médico del IMSS, Morelos, sólo está identificando a 1\% del total estimado de casos de mujeres maltratadas.

En relación con la actitud para canalizar a las mujeres maltratadas a los servicios especializados, la única variable significativa fue el sexo del personal médico, mientras que a la actitud general y a la actitud para identificar casos se asociaron, además del sexo, las variables mencionadas en los cuadros III y IV. El que la actitud para canalizar casos de mujeres maltratadas mostrara escasa consistencia, con relación a las otras actitudes, se debió probablemente a que ésta fue medida con sólo cuatro preguntas, mientras que la actitud general fue medida con 40 , y la actitud para identificar casos, con 17.

Al comparar los resultados de nuestro estudio con otros similares, respecto a la instrucción previa que tiene el personal médico sobre el tema, destacan los estudios realizados por Gremillion y Elaine J, en 1996 y 1998, respectivamente, ${ }^{33,34}$ en los EUA, en los que se encontró que cerca de $54 \%$ de las escuelas de medicina de ese país ofrecen programas de instrucción sobre violencia intrafamiliar, y que más de un tercio -de 1521

\footnotetext{
* Fawcett G, Venguer T, Vernon R, Pick S. Detección y manejo de mujeres víctimas de violencia doméstica: desarrollo y evaluación de un programa dirigido al personal de salud. México, DF: Population Council, INOPAL III, 1998;26:7-22. Documento de Trabajo.
}

practicantes clínicos de distintas disciplinas- nunca recibió instrucción sobre violencia. Otro trabajo realizado por Reid SA y Glasser M en 1997, ${ }^{18}$ en el oeste del mismo país, encontró que $75 \%$ de los médicos no han sido entrenados para enfrentar este problema. En México destaca el estudio de Díaz-Martínez y Esteban-Jiménez ${ }_{r}^{35}$ quienes exploraron la inclusión de contenidos temáticos de violencia intrafamiliar en diversas actividades académicas de 11 escuelas y facultades de medicina en el país. Los resultados muestran que $90 \%$ de las instituciones encuestadas sí imparten contenidos de violencia intrafamiliar. No obstante, más de la mitad aborda los temas de manera indirecta; esto es, sólo se les menciona o bien se incide en ellos de manera tangencial. Más aún, aquellas instituciones que los realizan de forma directa lo hacen a través de actividades extracurriculares como cursos, talleres, diplomados o actividades de difusión. En ningún caso se reportó una actividad que específicamente abordara los temas en el currículo. También quedó claro que la mayor parte de las instituciones carece de dependencias especializadas para su abordaje y tratamiento, y que los esfuerzos que se realizan parecen necesitar de una mayor orientación y apoyo.

Estudios previos muestran que más de $67 \%$ de las mujeres esperan que sea el personal médico quien les pregunte acerca de la violencia que sufren ${ }^{36} \mathrm{y}$ más de $60 \%$ del personal médico espera que la mujer maltratada sea quien dé la señal o consentimiento para abordar dentro de la consulta médica sus experiencias de violencia. ${ }^{23}$ En el presente estudio encontramos que más de $70 \%$ del personal médico piensa que la mujer maltratada es quien debe iniciar la plática sobre violencia.

Una vez que se ha identificado el caso, lo ideal es canalizarlo a instituciones y personal capacitado para que le brinde ayuda especializada; sin embargo, Fawcett reporta que $43.9 \%$ del personal médico de su estudio refiere la víctima al psicólogo del hospital y $36.6 \%$ al departamento de trabajo social. En nuestro estudio, más de la tercera parte del personal médico del IMSS, Morelos, sólo platica con las pacientes tratando de ayudarlas; otra tercera parte da tratamiento médico y $25 \%$ refieren los casos de mujeres maltratadas a instituciones o personal que proporcionan atención especializada; -aunque sólo 15\% logró mencionar alguna institución especializada que brinde atención a mujeres con este problema.

Referente a las barreras institucionales y de la práctica médica que dificultan la identificación y canalización de los casos de mujeres maltratadas encontramos lo siguiente: tanto en nuestro estudio como los de Valdez R, $2002^{12}$ y Alpert ${ }^{37}$ se observa que la falta de 
normas, protocolos de identificación y manejo, así como la escasa legitimidad social de las intervenciones del personal en este problema de salud, son las principales causas institucionales; situación que podría modificarse con la aplicación de la Norma Oficial Mexicana (NOM-190-SSA1-1999) promulgada en marzo del año 2000. No obstante, el aplicar esta norma no garantiza cambios en la manera de proceder del personal de salud, más bien, son la respuesta social, las tendencias ideológicas y políticas las que promueven los cambios. Respecto a las barreras de la práctica médica se observa que éstas se deben principalmente al desconocimiento sobre el tema y a la carencia de herramientas en los médicos para enfrentar estos casos. También existen algunos médicos que manifiestan falta de control y frustración ante la respuesta de la paciente, ya que desconocen el efecto de sus consejos. ${ }^{*}, 22$

En relación con la violencia que involucra al personal médico, Sugg e Inui $1992^{22}$ encontraron que 31\% de las médicas y $14 \%$ de los médicos reportaron padecer violencia por parte de su pareja; en nuestro estudio $21 \%$ de los entrevistados refieren padecer violencia, siendo más frecuente en las mujeres. A la vez, $16 \%$ del personal médico refiere ser autor de violencia hacia su pareja; lo cual muestra que el personal médico -al igual que cualquier persona- se encuentra inmerso en las mismas circunstancias socioculturales que determinan la desigualdad entre los géneros. Por ello, capacitar al personal médico en torno a la violencia es necesario, considerando que necesitan ayuda especializada para resolver sus propios conflictos, antes que los de sus pacientes.

Las médicas muestran mayor empatía y menos vergüenza para tratar el tema de la violencia dentro de la consulta, porque, al parecer, se sienten en mayor riesgo que los varones. Además, se ha observado que las pacientes revelan más información a las médicas; sin embargo, la mayoría del personal médico está constituido por varones, y por lo general éstos están menos interesados en el tema. ${ }^{38}$

Hasta el momento la violencia hacia la mujer es un tema que no ha sido abordado directamente por la enseñanza y práctica de la medicina, y uno de los aspectos que se requiere trabajar de manera inmediata es, precisamente, la escasa y equívoca información que

\footnotetext{
* Fawcett G, Venguer T, Vernon R, Pick S. Detección y manejo de mujeres víctimas de violencia doméstica: desarrollo y evaluación de un programa dirigido al personal de salud. México, DF: Population Council, INOPAL III, 1998;26:7-22. Documento de Trabajo.
}

el personal médico posee respecto del problema. En este sentido, sería enriquecedor profundizar en el aspecto del desconocimiento generalizado, pues no se satisfacen aún las necesidades de identificación, manejo y control de daños a la salud de las mujeres, secundarios a la violencia. Y, aunque se han realizado intervenciones -en EUA y Canadá- para tratar de mejorar los mecanismos de identificación, registro, atención en los servicios de salud, servicios sociales y fortalecimiento de leyes que protejan los derechos de las mujeres que sufren violencia, aún no se logran establecer programas y estrategias sólidas y efectivas..$^{39}$ En México se están evaluando cuáles son las necesidades de capacitación, y qué tipo de médicos y personal de salud respondería con mejores resultados a la capacitación en el tema, ya que se ha encontrado que las enfermeras y las trabajadoras sociales realizan más rutinariamente preguntas para identificar maltrato en las mujeres que acuden a los servicios de salud, en comparación con los médicos. ${ }^{40} \mathrm{El}$ presente estudio muestra que a pesar de que los médicos generales, los especialistas en medicina familiar y ginecobstetricia, los médicos con posgrado y las mujeres tienen mayor nivel de conocimiento, mejor actitud afectiva, identifican mayor número de casos y han recibido mayor cantidad de capacitaciones, aún no se identifica, registra y proporciona atención satisfactoria a las mujeres víctimas de violencia.

Con base en lo anterior quedan caras las limitaciones del enfoque biomédico para la intervención en los casos de violencia familiar, por lo que se justifica -a mediano y largo plazo-la incorporación de este tipo de temas, desde una perspectiva social, en los planes de estudio de licenciatura, posgrados o cursos de capacitación continua, tanto en el área médica como en otras relacionadas con la salud y los derechos humanos.

En este sentido, las intervenciones que se realicen al respecto deberán considerar como requisito indispensable el ofrecer un número suficiente de capacitaciones que permitan al profesional de la salud formarse un criterio sólido y claro con relación al papel que juegan éste y su práctica clínica en la identificación, registro, prevención y atención de los daños a la salud de las mujeres, secundarios a la violencia; ${ }^{41}$ además, considerar la integración del resto del personal de salud en los programas e intervenciones. No obstante, a pesar de que el entrenamiento a los profesionales de la salud ha mostrado incrementar considerablemente su conocimiento, habilidades ${ }^{42}$ y seguridad, cuando realizan actividades de identificación, también es sabido que sin cambios estructurales en las distintas instituciones involucradas en la atención a las víctimas de la violencia, así como la educación médica continua, es poco 
probable que sólo el entrenamiento sea suficiente para producir cambios importantes. ${ }^{31,43}$

Lo que se intenta es que reconozcan la magnitud, cualidades y repercusiones del problema, y la oportunidad que tienen para apoyar a las mujeres maltratadas en la búsqueda de soluciones a su problema, ya que, tarde o temprano, éstas acudirán a los servicios por algún problema de salud secundario a la violencia que sufren. Por último, resulta necesario considerar que el proceder del personal de salud tendrá impacto sobre el desempeño laboral y la calidad de vida de las mujeres que son maltratadas por su pareja.

\section{Referencias}

1. Heise L, Pitanguy J, Germain A.Violencia contra la mujer : la carga oculta sobre la salud.W ashington, DC: 0 rganización Panamericana de la Salud, 1994:1-48.

2. Flicraft AH, Hadley SM, Hendricks-Mattehews MK, McLeer SV, W arshaw C.American Medical Association Diagnostic and Treatment Guidelines on D omestic Violence. Arch Fam Med 1992;1:39-47. 3. Jaffe P, Suderman M, Schieck E. Programa escolar contra la violencia: Canadá. En: Morrison-Andrew R, Loreto-Biche M, ed. El costo del silencio.Violencia doméstica en las A méricas.W ashington, DC: Banco Interamericano de Desarrollo, 1999:161-183.

4. Rivera L. Prevalencia de violencia de género en el ámbito conyugal (tesis). Cuernavaca, Morelos, México: Instituto N acional de Salud Pública, 1998.

5. McFarlane J. Battering during pregnancy:Tip of an Iceberg revealed. Women Health 1989;15(3):69-84.

6. Saucedo-G onzález I.Violencia doméstica y salud: conceptualización y datos que existen en México. Perinatol Reprod Hum 1996;10:100-110. 7.Valdez R, Juárez C. Impacto de la violencia doméstica en la salud mental de las mujeres: análisis y perspectivas en México. Salud Mental 1998;21(6):1-10.

8. Gómez A.Violencia contra las mujeres: un ejercicio de poder. En: Por el derecho a vivir sin violencia. Acciones y Propuestas desde las mujeres. Cuernavaca, Morelos, México: C uadernos Mujer Salud/Red de salud de las mujeres latinoamericanas y del Caribe: 8,9, 123-138.

9. Plichta SB,A braham C.Violence and gynecologic health in women < 50 years old. Am J O bstet Gynecol 1996;174(3):903-907.

10.Valdez-Santiago R. Panorama de la violencia en México. En Valdez R, Hom P, comp.Violencia doméstica. Programa: educación y cultura, Cuernavaca, Morelos: Cidhol: Centro para Mujeres,1998:13-21. 11.Valdez R, Sanín LH. La violencia doméstica durante el embarazo y su relación con el bajo peso al nacer. Salud Publica Mex 1996;38:352-362. 12.Valdez R. Respuesta médica ante la violencia que sufren las mujeres embarazadas. En:Violencia contra la mujer en distintos contextos culturales. Programa interdisciplinario de estudios de la mujer (PIEM) del Colegio de México 2002. En prensa.

13. Banco Mundial. Informe sobre el desarrollo Mundial.W ashington, DC: Banco Mundial, 1993:7-10.

14. Lozano R. La carga de la enfermedad y las lesiones por violencia contra las mujeres: el caso de México. México, DF: Fundación Mexicana para la Salud, Centro de economía y salud, 1997.

15. Ley de asistencia y prevención de la violencia intrafamiliar. México, DF: Diario 0 ficial de la Federación, julio 1995.

16. Congreso de la Unión, decreto que reforma diversos artículos de los códigos civil, de procedimientos civiles, penal y de procedimientos penales. México, DF: Diario 0 ficial de la Federación, 27 de diciembre de 1997.

17. Secretaría de Salud. N orma O ficial Mexicana N O M-190-SSA 1-1999.

Prestación de servicios de salud. Criterios para la atención médica de la violencia familiar. México, DF: Diario 0 ficial de la Federación, 8 de marzo de 2000.

18. Reid SA, G lasser M. Primary care physicians' recognition of and attitudes toward domestic violence. A cademic Med 1997;72(1):51-53. 19. Ferris L. C anadian family physician's and general practitioners perceptions of their effectiveness in identifying and treating wife abuse. Med Care 1994;32(12):1163-1172.

20. Cohen S, D e Vos E, N ewberger E. Barriers to physician identification and treatment of family violence: Lessons from five communities. Academic Med 1997;72(Suppl 1):S19-S25.

21. Hartigan P. Ruta crítica de la violencia intrafamiliar. Lecciones del proyecto. Boletín informativo de la Red Feminista Latinoamericana y de El Caribe contra laViolencia Doméstica y Sexual. Isis Internacional, Chile, enero 1998:8-9.

22. Sugg N K, Inui T. Primary care physicians' response to domestic violence. 0 pening Pandora's Box. JA MA 1992;267(23):3157-3160. 23. Híjar MC, Lozano R,Valdez R, Blanco J. Lesiones intencionales como causa de demanda de atención en servicios de urgencia hospitalaria en la Ciudad de México. Salud Mental 2002;25(1):35-42.

24. Harwell TS, C asten RJ,Armstrong KA, Demsey S, C oons HL. Results of a domestic violence training program offered to the staff of urban community health centers. Evaluation Committee of the Philadelphia Family Violence W orking G roup. Am J Prev Med 1998;153:235-242. 25. D awis-Robin M. Fundamentos y técnicas de medición de las actitudes. México, DF: Editorial Limusa, 1983:29.

26. Blalock H. Medición de las actitudes. En: Introducción a la investigación social. Buenos Aires, Argentina:A morrortu Editores, 1998:98-129.

27. Casales JC. Estudio de las actitudes. En: Psicología social. Contribución a su estudio. La Habana: Editorial de Ciencias Sociales, 1989:158-199.

28. Moscovici S,A bric JC, Brehm S, D oise W, D oms M, Lerine JM et al. El cambio de actitud. En: psicología social. I. Influencia y cambio de actitudes, individuos y grupos. 5a ed. Barcelona, España: Ediciones Paidós, 1985:117-168.

29. Silva-Ayçaguer L. Excursión a la regresión logística en ciencias de la salud. Madrid, España: Ediciones Díaz Santos, 1995:3-10.

30. Hernández-Avila M, Garrido F, Salazar-Martínez E. Sesgos en estudios epidemiológicos. Salud Publica Mex 2000;42(5):438-446.

31. Rodríguez-Michael A, Bauer-Heidi M, McLoughlin E, Grumbach K. Screening and intervention for intimate partner abuse. Practice and attitudes of primary care physicians. JAMA 1999;282(5):468-474. 32. Parsons LH, Zaccaro D, W ells B, Stoval TG. Methods of and attitudes toward screening obsterics and gynecology patients for domestic violence. Am J O bstet Gynecol, 1995;173(2):381-387.

33. G remillion DH, Kanof EP. 0 vercoming barriers to physician involvement in identifying and referring victims of domestic violence. Ann Emerg Med 1996;27(6):769-773.

34. Alpert EJ, Allison E, Seeherman AM, HItz H. Family violence curricula in US Medical Schools. Am J Prev Med 1998;14(4):273-278.

35. Díaz-Martínez A, Esteban-Jiménez R. Enseñanza de contenidos de violencia intrafamiliar y sexual en instituciones de educación superior. Gac Med Mex 1999;135(3):274-281.

36. O Ison L,Anctil C, Fullerton L, Brillman J,Arbuckle J, Sklar D. Increasing emergency physician recognition of domestic violence. $A n n$ Emerg Med 1996;27(6):741-746.

37. Alpert EJ, Cohen S, Sege RD. Family violence:An overview. Acad Med 1997;72(Suppl 1):S3-S6.

38. Kellermann AL. D omestic violence and the internist's response: Advocacy or apathy? J G Intern Med 1990;5:89-90. 
39. Chalk R, King P.Assesing family violence interventions. Am J Prev Med 1998;14(4):289-292.

40. Gerbert B, Caspers N , Bronstone A, Moe J, A berecrombie P.A qualitative analysis of how physicians with expertise domestic violence approach the identification of victims.Ann Intern Med 1999;131: 578-584.

41. Chescheir $\mathrm{N}$,Violence against women: Response from clinicians. Ann Emerg Med 1996;27(6):766-768.
42. N ovack DH, Suchman AL, C lark W, Epstein R M, N ajberg E, kaplan $C$ raig et al. Calibrating the physician. Personal awareness and effective patient care. JAMA 1997;278(6):502-509.

43. González S. La violencia doméstica y sus repercusiones en la salud reproductiva en una zona indígena (C uetzalan, Puebla). En: Fundación McArthur/A sociación Mexicana de población, comp. Los silencios de la salud reproductiva: violencia, sexualidad y derechos reproductivos. México, DF: 1998:17-54. 